\title{
O programa teórico da residência em Medicina de Família e Comunidade do Serviço de Saúde Comunitária do Grupo Hospitalar Conceição, Porto Alegre, RS
}

\author{
The didactics component of the Family and Community Medicine Residency Program of the Community Health Service of the
} Grupo Hospitalar Conceição, Porto Alegre, RS

\section{El Programa Teórico de la Residencia en Medicina Familiar y Comunitaria del Servicio de Salud Comunitaria del Grupo Hospitalar Conceição, Porto Alegre, RS}

Rodrigo Caprio Leite de Castro. Serviço de Saúde Comunitária do Grupo Hospitalar Conceição (SSC/GHC). Universidade Federal do Rio Grande do Sul (UFRGS). Porto Alegre, RS, Brasil. rodrigo_caprio@yahoo.com.br (Autor correspondente)

Margarita Luz Marina Silva Diercks. Serviço de Saúde Comunitária do Grupo Hospitalar Conceição (SSC/GHC). Porto Alegre, RS, Brasil. margarita.diercks@yahoo.com.br

Felipe Anselmi Corrêa. Serviço de Saúde Comunitária do Grupo Hospitalar Conceição (SSC/GHC). Porto Alegre, RS, Brasil. felipeanselmi@gmail.com Ney Bragança Gyrão. Serviço de Saúde Comunitária do Grupo Hospitalar Conceição (SSC/GHC). Porto Alegre, RS, Brasil. gyral@terra.com.br Renata Pekelman. Serviço de Saúde Comunitária do Grupo Hospitalar Conceição (SSC/GHC). Porto Alegre, RS, Brasil. renatapek@gmail.com Roberto Nunes Umpierre. Serviço de Saúde Comunitária do Grupo Hospitalar Conceição (SSC/GHC). Universidade Federal do Rio Grande do Sul (UFRGS). Porto Alegre, RS, Brasil. rnumpierre@gmail.com

\section{Resumo}

A Sociedade Brasileira de Medicina de Família e Comunidade, baseando-se nas resoluções da Comissão Nacional de Residência Médica, recomenda que os Programas de Residência Médica em Medicina de Família e Comunidade (PRMMFC) dediquem de 10\% a 20\% de sua carga horária ao desenvolvimento de atividades teóricas. Destaca ainda que, para a elaboração do processo pedagógico dessas atividades, deve ser considerada a forma de aprendizado dos adultos, que é produzida com base nos problemas enfrentados no dia a dia. 0 Programa de Residência Médica em Medicina de Família e Comunidade do Serviço de Saúde Comunitária do Grupo Hospitalar Conceição (PRMMFC do SSC/GHC) tem por objetivo formar médicos para a Atenção Primária à Saúde, qualificados e resolutivos, com uma prática integradora e continuada, em equipe multidisciplinar, inserida nas comunidades sob seus cuidados. 0 presente artigo apresenta o Programa Teórico de Núcleo (PTN) do PRMMFC do SSC/GHC, que é a parte do Programa Teórico desta residência responsável pela formação dos aspectos específicos da Medicina de Família e Comunidade. Este relato tem como objetivo contribuir com a práxis metodológica das atividades teóricas dos PRMMFC no Brasil. São apresentados, inicialmente, o Serviço de Saúde Comunitária do Grupo Hospitalar Conceição, o PRMMFC do SSC/GHC e o Programa Teórico do PRMMFC do SSC/GHC. Em seguida, o PTN é relatado por meio dos seus quatro elementos constituintes: o eixo norteador, os eixos temáticos e os seus respectivos conteúdos, o processo pedagógico e a avaliação.

\begin{abstract}
The Brazilian Society of Family and Community Medicine, based on resolutions of the National Commission of Medical Residency, recommends that Family and Community Medicine Medical Residency Programs (FCMMRP) dedicate between 10\% and 20\% of their workload to didactics. It also highlights that, when designing the pedagogical process of these activities, adult learning must be considered, which occurs on the basis of the daily problems faced by them. The objective of the Family and Community Medicine Medical Residency Program of the Community Health Service of the Grupo Hospitalar Conceição (FCMMRP of CHS/GHC) is to form qualified and problem-solving physicians for Primary Health Care, with an integrative and continued practice, in multidisciplinary teams, inserted within the communities under their care. This paper introduces the Theoretical Core Program (TCP) of the FCMMRP of CHS/GHC, which is the section of the Didactics Program responsible, in this residency, for training in the aspects that are specific to Family and Community Medicine. This report aims to contribute with the methodological praxis of the didactic activities of the FCMMRP in Brazil. First the article introduces the Community Health Service of the Grupo Hospitalar Conceição, its Family and Community Medicine Medical Residency Program and the didactics component of this program. Next, the TCP is presented by means of its four constituent elements: guiding axis; thematic axis and its respective contents; pedagogical process; and evaluation.
\end{abstract}

Como citar: Castro RCL, Diercks MLMS, Corrêa FA, Gyrão NB, Pekelman R, Umpierre RN. O programa teórico da residência em Medicina de Família e Comunidade do Serviço de Saúde Comunitária do Grupo Hospitalar Conceição, Porto Alegre, RS. Rev Bras Med Fam Comunidade. 2014;9(33):375-383.. Disponível em: http://dx.doi.org/10.5712/rbmfc9(33)855.
Palavras-chave: Medicina de Família e Comunidade Desenvolvimento de Pessoal Educação Médica

Keywords: Family Practice Staff Development Education, Medical 


\section{Resumen}

La Sociedad Brasileña de Medicina Familiar y Comunitaria, basada en las resoluciones de la Comisión Nacional de Residencia Médica, recomienda que los Programas de Residencia Médica en Medicina Famíliar y Comunitaria (PRMMFC) tengan de 10\% a 20\% de su carga de trabajo para la realización de actividades teóricas. Destaca que el proceso pedagógico de estas actividades deben tener una pedagogia de adultos, que se caracteriza, entre otros aspectos, por discutir y analisar de manera crítica los problemas cotidianos enfrentados por los residentes. El Programa de Residencia Médica en Medicina Familiar y Comunitaria del Servicio de Salud Comunitaria del Grupo Hospitalar Conceição (PRMMFC del SSC/GHC) tiene como objetivo preparar y perfeccionar médicos para la Atención Primaria a la Salud. En este artículo se presenta el Programa Teórico de Núcleo (PTN) del PRMMFC del SSC/GHC, que es la parte del Programa Teórico, de esta residencia, responsable de la formación de los aspectos específicos de la Medicina Familiar y Comunitaria. Este artículo tiene como objetivo contribuir con la praxis metodológica de las actividades teóricas de los PRMMFC en Brasil. Describimos, inicialmente, el Servicio de Salud Comunitaria del Grupo Hospitalar Conceição, el PRMMFC del SSC/GHC y el Programa Teórico del PRMMFC del SSC/GHC. A continuación, la PTN es reportado por sus cuatro elementos constitutivos: el principio rector, los temas y sus respectivos contenidos, el proceso de enseñanza y la evaluación.
Palabras clave: Medicina Familiar y Comunitaria Desarrollo de Personal Educación Médica

\section{Introdução}

O Médico de Família e Comunidade (MFC) cuida da saúde das pessoas, famílias e comunidades de forma abrangente, continuada e contextualizada, tendo como cenário privilegiado de sua prática a Atenção Primária à Saúde (APS).

No Brasil, o Ministério da Saúde utiliza, para a expansão da APS e, portanto, para a reorientação do Sistema Único de Saúde (SUS), a Estratégia Saúde da Família (ESF). ${ }^{1} \mathrm{O}$ financiamento da ESF vem crescendo, tendo ultrapassado 3 bilhóes de reais por ano em 2012 e 2013 . Vem crescendo também o número de equipes implantadas, sendo que, em dezembro de 2013, já eram 34.715 equipes, cuidando de, aproximadamente, 109 milhóes de pessoas, o que corresponde a 56,4\% da população brasileira. ${ }^{2}$

Da mesma forma, vem crescendo, conjuntamente com a ESF, a necessidade de formação de Médicos de Família e Comunidade capacitados para atuar na Estratégia, sendo este, hoje, um dos grandes desafios para a consolidação de uma APS de qualidade. Anderson et al., ${ }^{3}$ em publicação referente à posição da Sociedade Brasileira de Medicina e Comunidade (SBMFC), em 2007, consideram que "a formação (do MFC) deve inspirar-se em um modelo de prática médica centrada na pessoa e na relação médico paciente, com foco na família e orientada para a comunidade, privilegiando, por consequência, o primeiro contato, o vínculo, a continuidade e a integralidade do cuidado na atenção à saúde."

Anderson et al., ${ }^{4}$ em artigo mais recente, ratificam a necessidade de se formarem Médicos de Família e Comunidade com conhecimentos, habilidades e atitudes centradas nas pessoas. Para isso, consideram que os Programas de Residência Médica (PRM) são o "padrão-ouro" para essa formação, pois não incluem somente uma suficiente carga horária de prática supervisionada, mas também a adequada orientação teórica. Destacam ainda que, no âmbito da APS, os Programas de Residência Médica em Medicina de Família e Comunidade (PRMMFC) são ainda mais relevantes se for levado em conta o desafio de formar profissionais que deverăo ocupar cada vez mais espaços de atividades docentes e de pesquisa.

O número de PRMMFC no Brasil vem aumentando nos últimos 10 anos, embora ainda tenhamos uma capacidade de formação inferior à necessidade de médicos com essa especialidade. Ademais, os PRMMFC existentes são heterogêneos com relação à prática supervisionada, tendo em vista as diferenças históricas, estruturais e processuais dos serviços e à formação teórica oferecida.

A respeito das atividades teóricas, os PRMMFC estruturam as suas respectivas atividades de acordo, não somente com o espaço e o tempo disponíveis no âmbito de cada Programa, mas também com o interesse e o conhecimento do corpo de preceptores e de residentes e com as necessidades de saúde locais e regionais.

Tendo em vista todas essas potenciais diferenças, a SBMFC, baseando-se nas resoluçôes da Comissão Nacional de Residência Médica (CNRM), ${ }^{5}$ recomenda que os PRMMFC dediquem de $10 \%$ a $20 \%$ de sua carga horária ao desenvolvimento de atividades teóricas, o que corresponde a uma carga horária semanal de 6 a 12 horas. ${ }^{3}$ De acordo com a SBMFC, as ferramentas de abordagem do MFC devem ser objeto desses espaços, incluindo "a pessoa, o ciclo de vida e a abordagem integral à saúde", "a família e os instrumentos de abordagem familiar" e "a comunidade e os instrumentos de abordagem comunitária", ressaltando que a obtenção de melhores resultados nas atividades teóricas depende do processo pedagógico utilizado. Sobre este último, a SBMFC destaca ainda que deve ser considerada a forma de aprendizado dos adultos, que é produzido com base nos problemas enfrentados no dia a dia, indicando, por fim, que a discussão de caso (de pessoas ou 
famílias), ou de situaçôes vivenciadas nos domicílios ou na comunidade, seguidos e/ou precedidos de leitura, pode ser uma boa opção para estruturar essas atividades.

O presente artigo apresenta o Programa Teórico de Núcleo do Programa de Residência Médica em Medicina de Família e Comunidade do Serviço de Saúde Comunitária do Grupo Hospitalar Conceição, que é a parte do Programa Teórico desta Residência responsável pela formação dos aspectos específicos da MFC. Este relato tem como objetivo contribuir com a práxis metodológica das atividades teóricas dos PRMMFC no Brasil.

\section{Descrição da experiência e discussão}

\section{Serviço de Saúde Comunitária do Grupo Hospitalar Conceição}

O Serviço de Saúde Comunitária (SSC) é constituído por 12 unidades de APS localizadas na zona norte de Porto Alegre. O SSC é vinculado ao Hospital Nossa Senhora da Conceição (HNSC), hospital integrante do Grupo Hospitalar Conceição (GHC), que é uma instituição pública federal.

No SSC desenvolvem-se o Programa de Residência Médica em Medicina de Família e Comunidade (PRMMFC do SSC), em atividade ininterrupta desde 1980, e o Programa de Residência Integrada em Saúde com ênfase em Saúde da Família e Comunidade (PRIS/SFC do SSC), este último, desde 2004, para profissionais das áreas de psicologia, odontologia, enfermagem e serviço social e, a partir de 2007, nas áreas de farmácia e nutrição. Para o primeiro, são abertas anualmente 22 vagas para residentes do primeiro ano e, para o segundo, 54.

As equipes do SSC são multiprofissionais e heterogêneas com relação ao número e à categoria profissional, sendo constituídas por médicos de família e comunidade, enfermeiros, odontólogos, psicólogos, assistentes sociais e agentes comunitários de saúde. Médicos internistas, pediatras e psiquiatras realizam apoio matricial, assim como nutricionistas e farmacêuticas. O SSC possui acesso direto a eletrocardiograma de esforço, endoscopia digestiva alta, colonoscopia, tomografia, ressonância magnética e exames de medicina nuclear, sendo esses exames realizados no HNSC. O acesso à atenção secundária se dá por meio da central de marcação de consultas da Secretaria Municipal de Saúde de Porto Alegre, com exceção dos ambulatórios de patologia cervical, de mastologia e de pré-natal de alto risco do HNSC, cujo acesso é direto. Possui também acesso direto à internação em uma enfermaria no HNSC com 18 leitos. Acerca da cobertura populacional, podemos afirmar que o SSC cobre, aproximadamente, 9\% da população de Porto Alegre (correspondendo a 120 mil pessoas).

\section{O PRMMFC do SSC}

O PRMMFC do SSC tem como objetivo formar médicos para a APS, qualificados e resolutivos, com uma prática integradora e continuada, em equipe multidisciplinar, inserida nas comunidades sob seus cuidados. ${ }^{6}$

A formação do médico residente é desenvolvida fundamentalmente em serviço, nas unidades de APS do SSC, integrando uma mesma equipe ao longo dos dois anos de residência. O primeiro ano da residência é composto de atividades nas unidades de APS, onde o médico residente realiza atendimento ambulatorial (compreendendo este $55 \%$ da carga horária), visitas domiciliares, grupos e outras atividades comunitárias e de educação em saúde, e de plantóes no HNSC (nas emergências clínica, pediátrica, cirúrgica e obstétrica). No segundo ano, mantém-se a ênfase nas atividades realizadas nas unidades de APS e incluem-se estágios em áreas necessárias à formação do MFC, tais como: medicina interna, abordagem familiar, dermatologia, saúde mental e gerenciamento. Além disso, no segundo ano, o médico residente dispóe de dois meses para realizar estágio optativo. Para concluir a residência, o médico residente deve realizar um projeto de pesquisa e entregar o relatório final, sendo este o Trabalho de Conclusão do Curso. ${ }^{6}$

O corpo docente é composto por preceptores especialistas em Medicina de Família e Comunidade nas unidades de APS, por especialistas focais das respectivas áreas de estágio em atenção secundária/terciária e por preceptores de outras profissões da saúde em algumas atividades teóricas e práticas, sendo o médico residente supervisionado em todas as suas atividades durante o período da residência. 


\section{0 programa teórico do PRMMFC do SSC}

O programa teórico do PRMMFC do SSC é desenvolvido ao longo dos dois anos de residência e tem como objetivo o ensino-aprendizagem dos conhecimentos necessários para a prática do MFC.

O programa é estruturado em dois grandes grupos de atividades, as de núcleo e as de campo, entendendo-se por núcleo, segundo o conceito de Campos, ${ }^{7}$ o conjunto de saberes e de responsabilidades específicos de cada profissão ou especialidade (no caso, da especialidade Medicina de Família e Comunidade) e, por campo, o conjunto de saberes e responsabilidades comuns ou confluentes das várias profissōes ou especialidades (no caso, das profissóes e suas respectivas especializaçôes atuantes em APS).

Desse modo, no PRMMFC do SSC, nas atividades de núcleo são abordados os conhecimentos e as práticas específicas da Medicina de Família e Comunidade e, nas atividades de campo, os conhecimentos e as práticas comuns a todas as profissóes da área da saúde que atuam em APS. O objetivo das atividades de campo é construir uma visão interdisciplinar sobre os problemas de saúde e as intervençôes e, para este fim, os residentes de Medicina de Família e Comunidade participam de atividades em conjunto com os residentes do PRIS/SFC do SSC.

Os conteúdos de núcleo do PRMMFC são desenvolvidos em quatro atividades distintas:

a) No Programa Teórico de Núcleo (PTN). O PTN é o espaço formador dos aspectos fundamentais da especialidade, sendo o objeto do presente artigo. Ele será abordado com detalhes no tópico seguinte.

b) Nas reunióes clínicas semanais nas unidades de APS. As reuniôes clínicas, que acontecem em todas as unidades de APS, têm como objetivo o ensino-aprendizagem da clínica mais prevalente no âmbito da Medicina de Família e Comunidade, sendo realizadas a partir de situações problema trazidas pelos preceptores ou de casos clínicos atendidos pelos residentes na unidade. São exemplos de assuntos vistos nessas reuniōes: hipertensão arterial sistêmica, diabetes mellitus, asma, puericultura, acompanhamento pré-natal, anticoncepção, depressão, ansiedade, etc.

c) Nas apresentaçóes de caso mensais do PRMMFC do SSC. As apresentaçóes de casos clínicos do Programa de Residência são realizadas pelos residentes, para todo o grupo de preceptores e colegas residentes.

d) Nas aulas expositivas sobre temas clínicos. As aulas expositivas sobre temas clínicos, que acontecem com periodicidade variável, consistem em aulas realizadas para todo o grupo de residentes, abordando assuntos pouco prevalentes na prática das unidades de APS e/ou de interesse comum para todas as unidades. São exemplos de assuntos vistos nessas aulas: saúde indígena, medicina rural, diagnóstico e manejo das situaçôes de urgência/emergência em APS, etc.

Os conteúdos de campo são vistos em duas atividades distintas:

a) Nos seminários de campo quinzenais nas unidades de APS. Os seminários de campo, que acontecem em todas as unidades de APS, têm como objetivo o ensino-aprendizagem dos conhecimentos e da prática interdisciplinar em APS, sendo realizadas a partir de situaçóes-problema trazidas pelos preceptores ou pelos residentes da unidade.

b) No Currículo Integrado (CI). O CI, com duração de três semestres, é o espaço de ensino-aprendizagem dos conhecimentos relativos ao campo da APS, sendo vistos, neste âmbito, os princípios e atributos da APS e as diversas ferramentas de abordagem populacional. No último semestre, os residentes elaboram um projeto de implementação e/ou qualificação da ESF em um pequeno município do interior do Rio Grande do Sul. ${ }^{8}$ Segue a mesma metodologia do PTN que será descrita no tópico seguinte.

\section{PTN: formação dos aspectos específicos da Medicina de Família e Comunidade}

O PTN é constituído por quatro elementos, a saber: o eixo curricular e sua categoria orientadora; os eixos temáticos e seus respectivos conteúdos; o processo pedagógico; e a avaliação. 


\section{Eixo curricular e categoria orientadora}

O eixo curricular de todo o processo de ensino-aprendizagem do PTN tem como categoria orientadora o cuidado integral. O cuidado integral, constituinte essencial da práxis do MFC, é produzido na relação médico-pessoa, em conjunto, portanto, com esta, sendo realizado de forma abrangente, longitudinal, contextualizada e no âmbito da equipe multiprofissional. A prática integral do MFC é exercida com empatia e resolutividade, levando em consideração os contextos subjetivos e familiares, de território e de trabalho. O cuidado integral perpassa todo o PTN, de maneira que todos os conteúdos e metodologias utilizadas convergem para o aperfeiçoamento e a qualificação do cuidado praticado pelo MFC.

\section{Eixos temáticos e os seus respectivos conteúdos}

O programa é dividido em três eixos temáticos. O primeiro chama-se "Encontro entre sujeitos: o médico e a pessoa"; o segundo, "O sujeito e seu contexto: a família e o ciclo vital" e o terceiro, "O sujeito e seu contexto: a família, a comunidade e o trabalho". No final do curso dos três eixos, a síntese dos conteúdos é realizada por meio da apresentação de "casos complexos" e da aprendizagem de método para supervisão ambulatorial. Observa-se que a definição dos eixos e dos seus respectivos conteúdos tem como objetivo aprofundar, de forma progressiva e dialética, a reflexão, compreensão e prática do residente em MFC.

O conteúdo do primeiro eixo temático é visto no primeiro semestre da residência e compreende o Método Clínico Centrado na Pessoa (MCCP), com os seus seis componentes:

1) explorando a doença e a experiência da doença;

2) entendendo a pessoa como um todo;

3) elaborando um plano conjunto de manejo dos problemas;

4) incorporando prevenção e promoção da saúde;

5) intensificando o relacionamento entre pessoa e médico; e

6) sendo realista.

O segundo eixo temático é desenvolvido no segundo semestre da residência e é constituído pelos seguintes conteúdos: a abordagem familiar pelo MFC, o ciclo de vida familiar e a abordagem de famílias no pré-natal, com o primeiro filho, com filho(s) adolescente(s) (incluindo a abordagem do adolescente), com doença crônica, com doença mental, com idosos, com idosos restritos ao leito e com cuidadores de pessoas em atenção domiciliar. São estudadas as ferramentas de genograma, P.R.A.C.T.I.C.E. e FIRO. Os conteúdos do terceiro eixo temático são vistos no terceiro semestre da residência e compreendem: a abordagem de famílias em situação de luto/morte e de vulnerabilidade, a identificação da rede de apoio e os aspectos relacionados com trabalho e saúde. A bibliografia e a filmografia utilizadas no PTN são apresentadas nos Quadros 1 e 2, respectivamente.

No final do terceiro semestre, cada residente apresenta um caso para os demais, seguindo o roteiro proposto por Stewart et al.," cujos itens estáo colocados no Quadro 3. A supervisão deste caso fica a cargo de outro residente, que deve fazê-la seguindo os passos do método de supervisão ambulatorial "preceptor em um minuto", ${ }^{10,11}$ descritos no Quadro 4. Esse exercício de apresentação de caso e supervisão tem por finalidade fazer a síntese de todos os conteúdos trabalhados no PTN.

É importante observar que, no segundo ano, o PTN se desenvolve em um semestre somente, pois durante o período dos estágios de medicina interna (dois meses) e optativos (dois meses) não se realizam atividades deste Programa. 
Quadro 1. Bibliografia utilizada no PTN: livros, protocolos do SSC e artigos.

\section{Livros utilizados no primeiro semestre}

McWhinney I, Freeman T. Manual de Medicina de Família e Comunidade. 3a ed. Porto Alegre: Artmed; 2010.

Stewart M, Brown J, Weston W, McWhinney I, McWilliam C, Freeman T. Medicina centrada na pessoa: transformando o método clínico. 2a ed. Porto Alegre: Artmed; 2010. Livros utilizados no segundo semestre

Watson W, McCaffery M, editors. Working with families: case-based modules on common problems in family medicine. Toronto: Department of Family \& Community

Medicine, University of Toronto; 2003.

Carter B, McGoldrick M. As mudanças no ciclo de vida familiar: uma estrutura para a terapia familiar. $2^{\mathrm{a}}$ ed. Porto Alegre: Artmed; 2001.

McGoldrick M, Gerson R, Petry S. Genogramas: avaliação e intervenção familiar. 3ª edição. Porto Alegre: Artmed; 2012.

Walsh F. Fortalecendo a resiliência familiar. São Paulo: Roca; 2005.

\section{Livros utilizados no terceiro semestre}

Watson W, McCaffery M, editors. Working with families: case-based modules on common problems in family medicine. Toronto: Department of Family \& Community

Medicine, University of Toronto; 2003.

Carter B, McGoldrick M. As mudanças no ciclo de vida familiar: uma estrutura para a terapia familiar. $2^{\mathrm{a}}$ ed. Porto Alegre: Artmed; 2001.

Sluzki C. A rede social na prática sistêmica: alternativas terapêuticas. 3ª ed. São Paulo: Casa do Psicólogo; 2006.

Protocolos do SSC

Conteúdo trabalhado

Lenz M, Flores R, editors. Atenção à saúde da criança de 0 a 12 anos. Porto Alegre: Abordagem de famílias com primeiro filho/filhos pequenos.

Editora do Hospital Nossa Senhora da Conceição; 2009.

Lenz M, Flores R, editors. Atenção à saúde da gestante em atenção primária à

saúde. Porto Alegre: Editora do Hospital Nossa Senhora da Conceição; 2011.

Ferreira S, Bianchini I, Flores R. A organização do cuidado às pessoas com

hipertensão arterial sistêmica em serviços de atenção primária à saúde. Porto

Alegre: Editora do Hospital Nossa Senhora da Conceição; 2011.

Ferreira S, Bianchini I, Flores R. A organização do cuidado às pessoas com diabetes

mellitus tipo 2, em serviços de atenção primária à saúde. Porto Alegre: Editora do

Hospital Nossa Senhora da Conceição; 2011.

\section{Artigos}

Pôrto A. A vida inteira que podia ter sido e que não foi: trajetória de um poeta

tísico. Hist Ciênc Saúde-Manguinhos. 2000;6(3):523-550. Disponível em:

http://dx.doi.org/10.1590/S0104-59702000000400003.

Rebelo L. Genograma familiar: 0 bisturi do médico de família. Rev Port Clin Geral.

2007;23:309-317.

Pereira M, Filgueiras M. A dependência no processo de envelhecimento: uma

revisão sobre cuidadores informais de idosos. Rev APS. 2009;12(1):72-82.

Conteúdo trabalhado

Abordagem de famílias no pré-natal.

Abordagem de famílias com doença crônica (hipertensão arterial sistêmica).

Abordagem de famílias com doença crônica (diabetes mellitus).

Conteúdo trabalhado

Componente 1 do MCCP: Explorando a doença e a experiência da doença.

Abordagem familiar pelo MFC. Estudo da ferramenta genograma.

Abordagem de famílias com idosos restritos ao leito e com cuidadores de pessoas em atenção domiciliar.

Quadro 2. Filmografia utilizada no PTN do PRMMFC do SSC.

\begin{tabular}{|l|l|l|}
\hline \multicolumn{2}{|c|}{ Filmes } & \multicolumn{2}{c|}{ Conteúdo trabalhado } \\
\hline Minha vida sem mim & Isabel Coixet. & Componente 1 do MCCP: Explorando a doença e a experiência da doença. \\
Janela da alma & João Jardim e Walter Carvalho & Componente 1 do MCCP: Explorando a doença e a experiência da doença. \\
Golpe do destino & Randa Haines & Componente 5 do MCCP: Intensificando o relacionamento entre pessoa e médico. \\
0 filho da noiva & Juan José Campanella & Abordagem de famílias com doença crônica. \\
Mar adentro & Alejandro Amenábar & Abordagem de famílias em situação de luto/morte. \\
0 quarto do filho & Nanni Moretti & Abordagem de famílias em situação de luto/morte. \\
\hline
\end{tabular}

Quadro 3. Apresentação de caso centrada na pessoa.

\begin{tabular}{|ll|}
\hline$N^{\circ}$ & \multicolumn{1}{c|}{ Itens para a apresentação de caso centrada na pessoa } \\
\hline 1 & A principal preocupação ou solicitação da pessoa. \\
2 & Experiência da pessoa sobre estar doente, comentários da pessoa, sentimentos, ideias, efeitos em seu funcionamento, expectativas, significado da doença. \\
3 & Doença: histórico da doença ou problema atual, história médica anterior, revisão dos sistemas, exame físico, exames laboratoriais, etc. \\
4 & Pessoa: perfil da pessoa, fase do ciclo de vida. \\
5 & Contexto: próximo (história familiar, genograma) e distante (cultura). \\
6 & Relacionamento pessoa-médico, questões de transferência e contratransferência, estabelecimento de uma base em comum (problemas, metas e papéis). \\
7 & Avaliação (lista de problemas). \\
8 & Discussão do caso. \\
9 & Plano de manejo proposto. \\
\hline
\end{tabular}

Fonte: Adaptado de Stewart et al., 2010.9 
Quadro 4. Passos do método de supervisão ambulatorial "preceptor em um minuto".

\begin{tabular}{|c|c|c|}
\hline $\mathrm{N}^{\circ}$ & Passos do método "preceptor em um minuto" & Exemplos \\
\hline 1 & Estabeleça o compromisso. & "0 que você acha que está acontecendo com este paciente?" \\
\hline 2 & Investigue as evidências de apoio. & "Como você chegou a esta conclusão?" \\
\hline 3 & Providencie retroalimentação positiva. & "Você realizou um ótimo trabalho nos aspectos x, y, z, etc." \\
\hline 4 & Ensine regras gerais. & "Sempre que você vir isso considere..." \\
\hline 5 & Corrija erros. & "A próxima vez que isso acontecer tente ou considere..." \\
\hline
\end{tabular}

Fonte: Adaptado de Neher J0 et al., 2003,10 Parrott S et al., 2006. ${ }^{11}$

\section{Processo pedagógico}

Para o adequado desenvolvimento dos conteúdos e da práxis do MFC, buscou-se um referencial pedagógico que necessariamente considerasse as vivências, os interesses, as dificuldades e as contradiçóes do residente em Medicina de Família e Comunidade na sua prática cotidiana. Nesse processo de ensino-aprendizagem requerido, experiencia-se uma aproximação entre a Aprendizagem Baseada em Problemas, ou Problem-Based Learning (PBL), ${ }^{12}$ e os princípios da problematização de Freire, ${ }^{13,14}$ duas linhas pedagógicas de referenciais teóricos diferentes, mas com vários consensos observados na prática do processo educativo. ${ }^{15}$

A aproximação dessas linhas pedagógicas permite que o residente realize, a partir da sua realidade/vivência, uma reflexão crítica do seu processo de aprendizado/trabalho e retorne para o seu cotidiano com aspectos enriquecedores da prática. No PTN, utiliza-se a problematização de Freire, segundo a metodologia resumida por Zanotto e De Rose:16 "a ação de problematizar acontece a partir da realidade que cerca o sujeito; a busca de explicação e solução visa a transformar a realidade, pela ação do próprio sujeito (sua práxis)". Esse processo é corroborado pelos inúmeros estudos que afirmam que o adulto está mais propenso a aprender quando ele experimenta uma necessidade de conhecer algo ou adquirir novas capacidades para conseguir fazer algo relevante para sua prática. ${ }^{12}$

Da mesma forma como acontece no PBL, em que os problemas são elaborados por uma equipe de especialistas, e, também, na problematizaçáo de Freire, em que os problemas são extraídos da realidade pela observação realizada pelos alunos, no PTN, as situações problema são casos clínicos fictícios trazidos pelos preceptores-facilitadores ou, na maioria das vezes, casos clínicos trazidos pelos residentes (casos atendidos pelos residentes nas unidades de APS e relacionados com o conteúdo a ser estudado).

Em ambas as metodologias, o problema (ou situação-problema/caso clínico) é o ponto de partida e o fio condutor do processo de ensino-aprendizagem. Os conteúdos de cada eixo temático são vistos por meio das situaçóes-problema, de forma que as mesmas se inter-relacionam e são vistas de forma sequencial e com um aprofundamento progressivo conforme $o$ andamento do semestre.

A aquisição de conhecimentos acontece na medida em que os residentes defrontam-se com o problema e buscam compreendê-lo ou solucioná-lo. É no pequeno grupo (PG) que a análise do problema é aprofundada. A formatação em PG facilita, por sua vez, a criação de ambiente favorável para o desenvolvimento do processo educativo, em especial no que diz respeito à participaçáo efetiva de todos, à cooperação e à construçáo conjunta de conhecimento. As metodologias do PBL e da problematização de Freire são ativas, no sentido em que consideram os conhecimentos de que o residente já dispóe. São também interativas, centradas no aluno e autodirecionadas, cabendo ao residente um papel expressivo na definição do que ele precisa aprender e o que/como fará para alcançar isso. Inclusive, nesse ponto, destaca-se que os residentes são sempre estimulados a realizar pesquisa suplementar, com o objetivo de buscar outras literaturas que náo as recomendadas pelo PTN, sendo esse item parte de sua avaliação semestral individual.

As atividades teóricas do PTN são desenvolvidas em pequenos grupos, de 6 a 8 residentes, mediadas por facilitador especialista em MFC e médico/preceptor do SSC; e em grande grupo (GG), formado pelo encontro de todos os pequenos grupos. Os preceptores-facilitadores participam em todas as fases de planejamento, organização, execução e avaliação do PTN. Dentre as técnicas utilizadas em PG, além do estudo a partir de situaçóes problema, destacam-se ainda a dramatização (role playing) e a filmagem de consultas (no PTN, todos os residentes, no primeiro semestre de curso, devem filmar uma consulta, apresentá-la e analisá-la em PG, seguindo-se, para isso, as orientaçôes apresentadas no livro de Stewart el al. ${ }^{9}$ ).

Os encontros em GG, com 2 horas de duração, visam a abrir (inaugurar) um novo tema ou fechar (sintetizar) um tema que já vem sendo trabalhado. Para esses encontros, utilizam-se de metodologias variadas e geralmente realizadas com profissionais convidados. O PG reúne-se uma vez por semana e tem duração de 2 hs. O GG acorre, em média, uma vez por mês. 


\section{Avaliação}

A avaliação no PTN acontece semestralmente, em três enfoques de interesse, sendo eles:

1) Avaliação dos conteúdos aprendidos pelos residentes:

1.1) No final do primeiro semestre, é solicitado a cada residente que escolha um caso e o relate, considerando a sua abordagem e todos os conteúdos vistos a respeito do MCCP ao longo do semestre, de forma a realizar uma apreciação crítica do cuidado praticado.

1.2) Ao longo do segundo semestre, é solicitado a cada residente que apresente um genograma de uma família acompanhada, com a devida análise de estrutura, dinâmica e de ciclo de vida familiar, além de um plano de abordagem.

1.3) No final do terceiro semestre, cada residente apresenta um caso para os demais, em PG, e a supervisão é realizada por outro colega residente, utilizando o método de supervisão ambulatorial "preceptor em um minuto", conforme apresentado no item "eixos temáticos e os seus respectivos conteúdos", sendo essa atividade considerada a última avaliação de conteúdo do PTN.

2) Avaliação de assiduidade, pontualidade, interesse, comprometimento e participação do residente. Essa avaliação é realizada no final de cada semestre, com cada residente, individualmente.

3) Avaliação do processo pedagógico e dos preceptores-facilitadores. Também no final de cada semestre, os residentes, individualmente e por escrito, avaliam o PTN: os aspectos a serem conservados, modificados ou suprimidos, as possíveis contribuiçóes do PTN para a formação profissional, as atividades em PG e em GG e a atuação do facilitador, além de uma autoavaliação.

\section{Conclusão}

O PTN iniciou em março de 2007 e está sendo continuamente avaliado pelos residentes, pelos preceptores-facilitadores e pelo colegiado da preceptoria (formado por todos os preceptores do PRMMFC).

Os preceptores-facilitadores são todos médicos de família e comunidade, assumindo-se como condição, para que o preceptor seja também facilitador do PTN, o acompanhamento de um PG por um período de 3 a 6 meses. Os preceptoresfacilitadores reúnem-se periodicamente para o planejamento e avaliação do PTN.

A literatura sobre educação médica refere-se insistentemente à necessidade de um médico capaz de conduzir, de forma autônoma, um processo de aprendizagem ao longo de sua vida profissional, de adaptar-se e participar das mudanças e que esteja apto também para raciocinar criticamente, para analisar sistemática e logicamente os problemas e tomar decisóes fundamentadas em sua própria avaliação.

A forma como é sistematizado o processo pedagógico do PBL e da problematização, destacando-se aqui a questão das situaçóes-problema vivenciadas e trazidas pelos residentes, assim como o trabalho em PG, propicia uma proposta pedagógica ativa, tendo como objetivo uma prática mais qualificada, reflexiva e autônoma.

O currículo do PTN, sistematizado pelo cuidado integral e sustentado por um processo pedagógico problematizador, à medida que aprofunda os conteúdos estruturantes da prática do MFC, provoca no residente a necessidade contraditória de desconstruir os conceitos e as práticas estabelecidas durante o curso de medicina, onde a fragmentaçáo do conhecimento desenvolvido na universidade segue, em geral, o referencial biomédico. Assim, a escolha do cuidado integral como categoria orientadora do PTN assegura a permanência, durante todo o curso do PTN, do desafio de construir a integralidade e a humanização do cuidado.

\section{Agradecimento e homenagem}

Agradecemos ao Dr. Luiz Felipe Cunha Mattos (in memoriam) por sua fundamental contribuição na elaboração e no aperfeiçoamento do Programa Teórico de Núcleo (PTN) do PRMMFC do SSC/GHC, ao longo de 4 anos (de 2007 até 2010), por meio de dedicado trabalho como preceptor-facilitador do PTN. 


\section{Referências}

1. Ministério da Saúde (BR). Saúde da família: uma estratégia para a reorientação do modelo assistencial. Brasília: Ministério da Saúde; 1998.

2. Sala de apoio à gestão estratégica (SAGE). Equipes da Saúde da Família [Internet]. Brasília: Ministério da Saúde; 2014 [acesso em 2014 Apr 23]. Disponível em: http://189.28.128.178/sage/.

3. Anderson MIP, Castro Filho ED, Rodrigues RD, Dalla MDB, Bourget MMM. Bases para expansão e desenvolvimento adequado de programas de Residência em Medicina de Família e Comunidade. Rev Bras Med Fam Comunidade [Internet]. 2007 [acesso em 2014 Apr 23];3(11):180-98. Disponível em: http://www.rbmfc.org.br/rbmfc/article/view/337.

4. Anderson MIP, Rodrigues RD. Formação de especialistas em Medicina de Família e Comunidade no Brasil: dilemas e perspectivas. Rev Bras Med Fam Comunidade [Internet]. 2011 [acesso em 2014 Apr 23];6(18):19-20. Disponível em: http://dx.doi.org/10.5327/Z1809-59092011001800005.

5. Ministério da Educação (BR). Comissão Nacional de Residência Médica (CNRM). Resolução 02/2006, de 17 de maio de 2006. Dispõe sobre requisitos mínimos dos Programas de Residência Médica e dá outras providências. Diário Oficial da União. 2006 May 19;95 Seção 1:23-36. Disponível em: http://portal.mec.gov.br/dmdocuments/resolucao02_2006.pdf.

6. Grupo Hospitalar Conceição, Hospital Nossa Senhora da Conceição, Serviço de Saúde Comunitária. Programa de Residência Médica em Medicina de Família e Comunidade. Porto Alegre: Grupo Hospitalar Conceição; 2013.

7. Campos GSW. Subjetividade e administração de pessoal: considerações sobre modos de gerenciar trabalho em equipe de saúde. In: Onoko R, Merhy E, editors. Agir em Saúde: um desafio para o público. São Paulo: Hucitec; Buenos Aires: Lugar Editoral; 1997.

8. Diercks MS, Pekelman R, Medeiros RHA, Silveira LR, Torres AA, Wilhelms DM, et al. O currículo integrado como estratégia de formação teórica em atenção primária à saúde para residentes dos programas de saúde da família e comunidade. In: Fajardo A, Rocha C, Pasini V, editors. Residências em Saúde: fazeres e saberes na formação em saúde. Porto Alegre: Editora do Hospital Nossa Senhora da Conceição; 2010. p. 173-190. Disponível em: http://escola.ghc.com.br/images/Publicacao/residenciaemsaude.pdf

9. Stewart M, Brown JB, Weston WW, McWhinney IR, McWilliam CL, Freeman TR. Medicina centrada na pessoa: transformando o método clínico. $2^{\text {a }}$ ed. Porto Alegre: Artmed; 2010.

10. Neher JO, Stevens NG. The one-minute preceptor: shaping the teaching conversation. Fam Med. 2003;35(6):391-3.

11. Parrott S, Dobbie A, Chumley H, Tysinger JW. Evidence-based office teaching - the five-step microskills model of clinical teaching. Fam Med. 2006;38(3):164-7.

12. Mamede S, Penaforte JC. Aprendizagem baseada em problemas: características, processos e racionalidade. Fortaleza: Hucitec; 2001.

13. Freire P. Extensão ou comunicação? Rio de Janeiro: Paz e Terra; 1977.

14. Freire P. Pedagogia da autonomia: saberes necessários à prática educativa. Rio de Janeiro: Paz e Terra; 1996.

15. Berbel NAN. A problematização e a aprendizagem baseada em problemas: diferentes termos ou diferentes caminhos? Interface (Botucatu). 1998;2(2):139-154. Disponível em: http://dx.doi.org/10.1590/S1414-32831998000100008.

16. Zanotto MAC, De Rose TMS. Problematizar a própria realidade: análise de uma experiência de formação contínua. Educ Pesqui. 2003;29(1):45-54. Disponível em: http://dx.doi.org/10.1590/S1517-97022003000100004. 